\title{
A Study of an Aero-Elastic Twisted Inverted Wing in Close Ground Proximity
}

\author{
Jean-Baptiste Barzic, ${ }^{1}$ Kevin Garry, ${ }^{2}$ and Simon Prince. ${ }^{3}$ \\ Applied Aerodynamics Group, School of Aerospace, Transport and Manufacturing \\ Cranfield University BEDFORD. UK
}

\begin{abstract}
Aero-elastic deformation of an inverted wing in close ground proximity can have an effect on the aerodynamic loads experienced and the downstream trajectory of the primary tip vortex. These differences have practical application to the front wing installations used in typical open-wheel auto-sport vehicle configurations. A series of moving ground wind tunnel measurements of aerodynamic lift and drag on a simplified inverted-wing-body configuration, at $\mathrm{Re} / \mathrm{m}$ in the range $1.4 \times 10^{6}<\mathrm{Re} / \mathrm{m}<2.8 \times 10^{6}$, were compared with $\mathrm{RANS}$ CFD simulations on both the un-deflected and deformed body. Measurements of model deformation were obtained using a videogrammetry technique. RANS CFD simulations appear to over-estimate the magnitude of the (-ve) lift coefficient, in very close ground proximity, compared to the experimental data. This may possibly be due to difficulty capturing the interaction between the wing tip vortex and a region of flow separation near the trailing edge, which is particularly apparent at low ground clearances.
\end{abstract}

\section{Nomenclature}

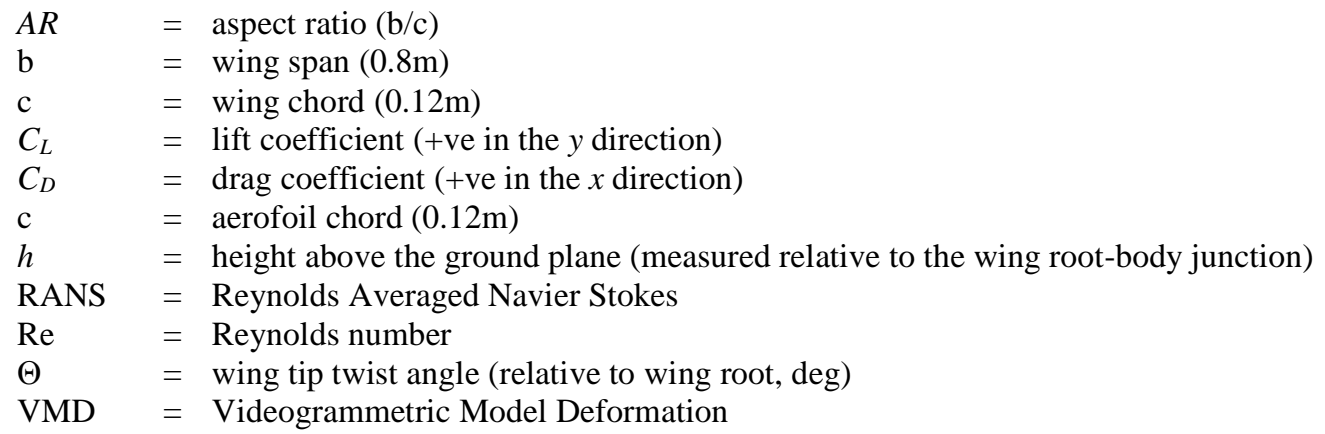

\section{Introduction}

Low aspect ratio, near rectangular planform inverted wings are routinely used in auto-sport applications to generate "negative lift" to enhance vehicle performance. Such configurations often operate in very close ground proximity and experience significant aerodynamic loads. There is potential to link the aero-elastic response of such wings to the required aerodynamic load characteristics, in order to optimize performance. Modelling this interaction - in very close ground proximity - requires insight into the changes induced in the primary flow features as a result of changes in span wise bending and twist.

\footnotetext{
${ }^{1}$ Aerospace Dynamics MSc Candidate.

${ }^{2}$ Professor of Experimental Aerodynamics. Member AIAA

${ }^{3}$ Senior Lecturer.
} 


\section{Background and Methodology}

In order to investigate the aero-elastic coupling a preliminary series of wind tunnel measurements and RANS CFD simulations were undertaken. The simplified geometry chosen for this study comprised a streamlined, flat-bottomed body with a rectangular planform inverted wing mounted from the lower forward section. The wing $(\mathrm{span}(\mathrm{b})=0.8 \mathrm{~m}$, aspect ratio $(A R)=6.67)$, has a NACA23012 profile section with chord $(c)$ of $0.12 \mathrm{~m}$. The wing is interchangeable on the body such that wings with span wise, zero-load, linear wash-in twist of $0,3,6$ and 8 degrees can be investigated at a range of pre-set wing-root angles of attack.
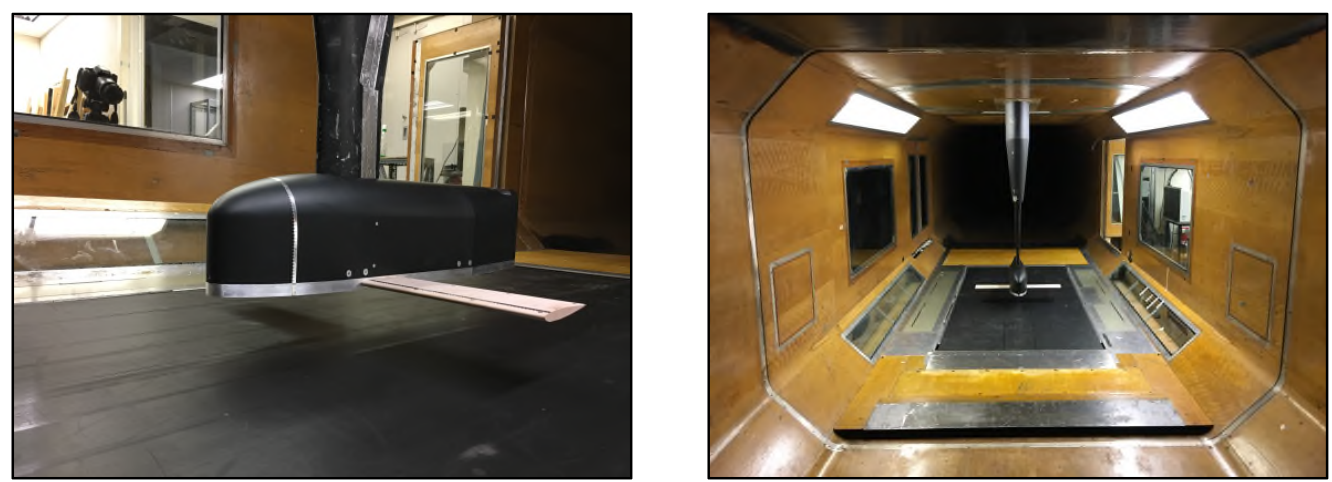

Figure 1. Simplified wing-body model mounted in the $8 x 6$ moving ground wind tunnel

Wind tunnel measurements were carried out in a $2.4 \mathrm{~m} \times 1.8 \mathrm{~m}$ closed working section, closed return facility with a moving ground plane to simulate ground proximity. The model is mounted from a single overhead main strut and tail strut assembly which facilitates remote adjustment of body pitch angle and ground clearance (h) in the range $0.05<$ $(\mathrm{h} / \mathrm{c})<0.76$. Measurement of aerodynamic forces and moments was made using an internal six-component strain gauge balance, at freestream Reynolds numbers in the range $1.40 \times 10^{6}<\mathrm{Re} / \mathrm{m}<3.24 \times 10^{6}$, both transition-free and transition-fixed (at $\mathrm{x} / \mathrm{c}=0.1$ ). An assessment of wing tip vortex trajectory was made using a grid of mono-filament tufts mounted at various axial downstream locations above the moving ground plane, viewed from downstream with a CCD camera. Direct comparison between the measured and predicted aerodynamic data is limited, in this instance, to lift coefficient in view of the relatively weak repeatability seen in the wind tunnel drag measurements.

Aerodynamic distortion of the wing under load was measured using a Videogrammetric Model Deformation (VMD) technique, see Barrows[1], Burner \& Liu[3]. This discrete non-intrusive method utilises observations, from a single fixed viewing point, of specific targets located on the wing surface. A series of preliminary investigations were undertaken to optimise the method in terms of camera position (viewing angle, distances, etc.) in view of the wind tunnel constraints imposed by the moving ground and the requirement to position the camera outside of the working section, taking images through a glass window. Bespoke MATLAB post-processing routines were devised to provide wing span-wise bending and twist data.

A parallel CFD study was undertaken using a RANS simulation in ANSYS Fluent vr17.1 to determine whether this approach could be used to predict the flow field with sufficient fidelity to enable it to be used for optimisation of more complex geometries. A 3D hybrid mesh was constructed with a $\mathrm{y}^{+}=3$ and 1.52 million cells following a mesh refinement study which compared 4 mesh sizes in the range 0.31 to $6.7 \mathrm{M}$. Mesh refinement recognised the need to avoid cell skewness at low ground clearances. Both the k- $\omega$ SST and Spalart-Allmaras (SA) turbulence models were initially investigated, the latter being selected for the majority of the simulations as it showed faster convergence with negligible difference between the predicted coefficients. 


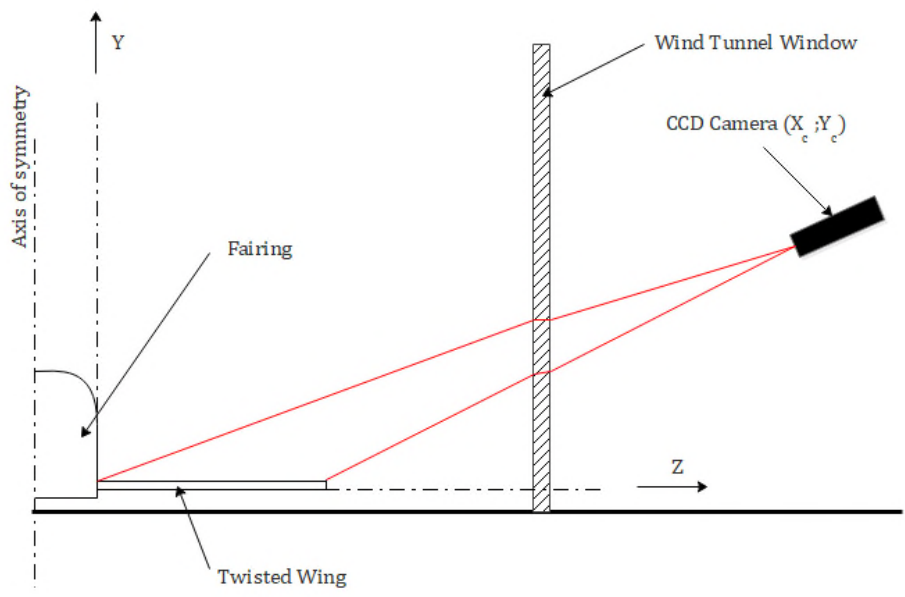

Figure 2. Schematic layout of the Videogrammetric Model Deformation (VMD) system adopted to measure wing span wise bending and twist.

\section{Summary of Observations}

Analysis of the predictions from the RANS CFD simulations, on the un-deflected (rigid) wing, shows that the general trend is for both (-ve) lift coefficient and drag coefficient to increase as the wing-body approaches the ground, see Fig 3. This effect becomes significant when non-dimensional ground clearance $(\mathrm{h} / \mathrm{c})<0.4$ and was as expected from previously published results, see for example Zerihan et al [8] and Zhang et al [7]. Increasing wing twist is also seen to increase both (-ve) lift and drag, see Fig 4. Reducing the ground clearance is seen to: (i) slightly reduce the effect of a change in twist on (-ve) lift coefficient and (ii) to noticeably increase the effect of twist on drag coefficient. Increasing twist therefore has an adverse effect on body lift-drag ratio at ground clearances below $(\mathrm{h} / \mathrm{c}) \approx 0.2$, see Fig 5 .
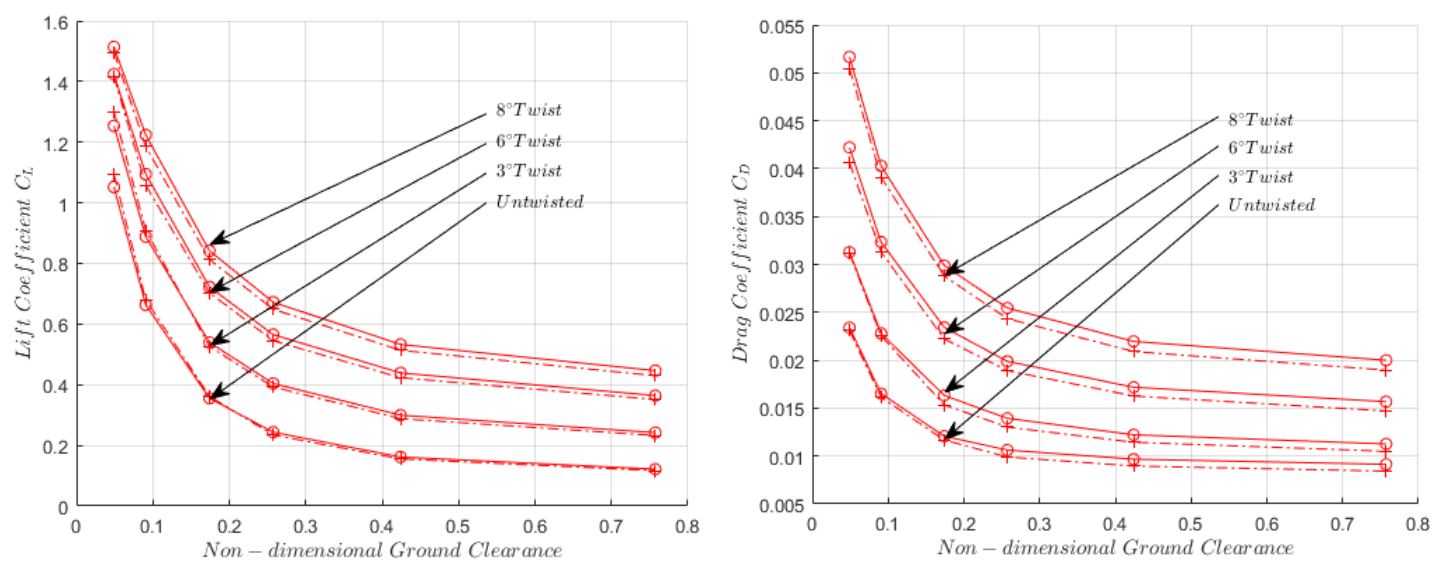

Figure 3. Variation of (-ve) lift coefficient and drag coefficient with non-dimensional ground clearance (h/c) for each of the four wing twists considered (broken line: $R e / m \approx 2.8 \times 10^{6}$, solid line: $R e / m \approx 1.4 \times 10^{6}$ ). 

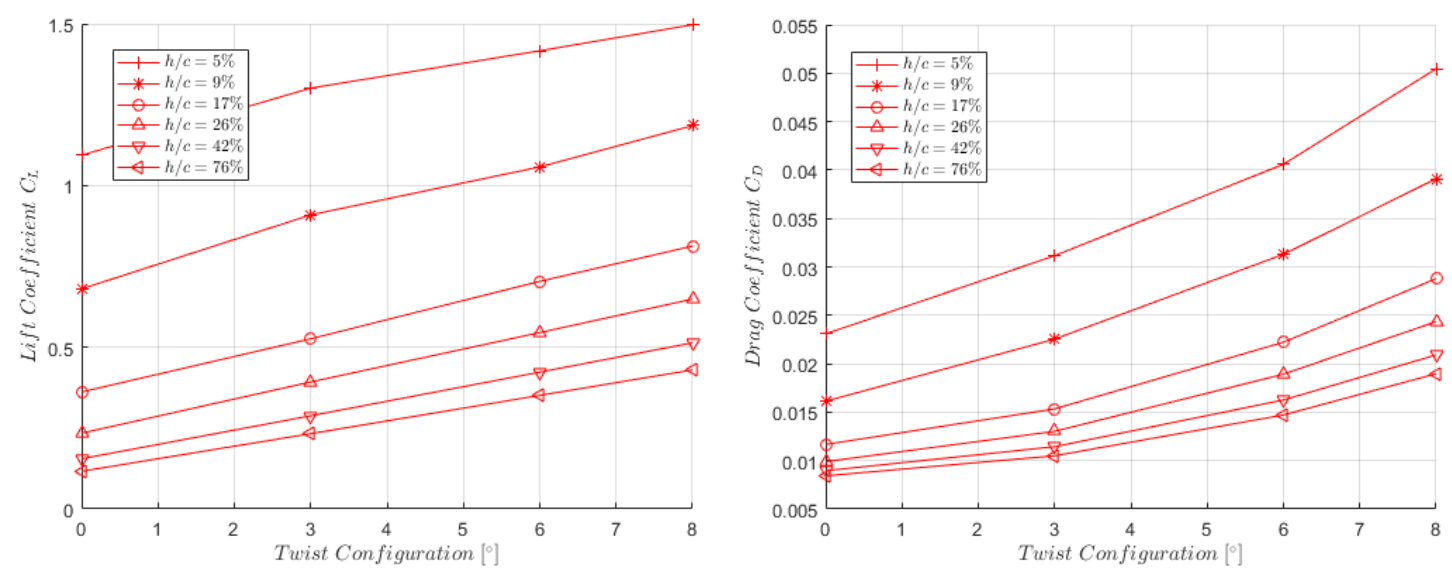

Figure 4. The effect on (-ve) lift and drag coefficient of changes in non-dimensional ground clearance $(\mathrm{h} / \mathrm{c})$ for a wing with linear spanwise twist of 8 degrees.

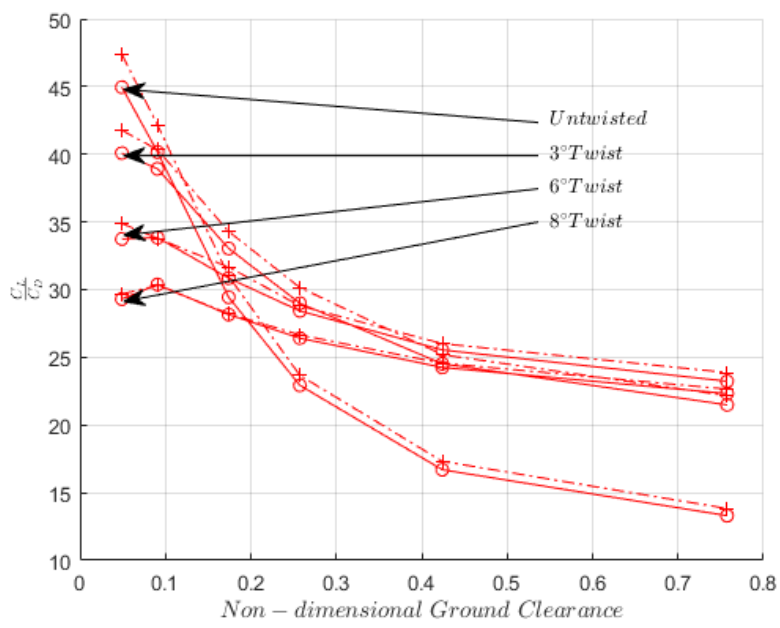

Figure 5. The influence of changes in non-dimensional ground clearance (h/c) on wing-body lift-to-drag ratio for each of the four wing twists considered (broken line: $R e / m \approx 2.8 \times 10^{6}$, solid line: $R e / m \approx 1.4 \times 10^{6}$ ).

The results from the wind tunnel experiments show slightly different variations with changes in non-dimensional ground clearance $(\mathrm{h} / \mathrm{c})$, depending on whether boundary layer transition was fixed or free in the wind tunnel tests, see Figures 6 and 7. In general, free transition results in a larger (-ve) lift coefficient compared with fixed transition conditions. Fixing transition adversely affects lift coefficient, particularly at low ground clearances and for high twist configurations, see Fig 6. For the case of the $8^{\circ}$ twisted wing (with fixed transition), two regions within the range of non-dimensional ground clearances tested, can be identified: (i) from out of ground effect to $(\mathrm{h} / \mathrm{c}) \approx 17 \%$, lift increases at an increasing rate, (ii) from $(\mathrm{h} / \mathrm{c})=17 \%$ to $10 \%$, lift increases at a decreasing rate. The ground clearance $(\mathrm{h} / \mathrm{c})$ at which the rate of increasing lift is a maximum $\left(17 \%\right.$ at $8^{\circ}$ twist $)$ is designated $\left(\mathrm{h}_{\text {maxrate }}\right)$, the magnitude of which reduces with decreasing tip twist. This means that the ground clearance at which a wing produces its maximum (-ve) lift coefficient ( $\mathrm{h}_{\text {maxlift }}$ ) occurs closer to the ground as wing tip twist is reduced. Increasing the test Reynolds Numbers (in this case through increased free stream dynamic pressure) is seen to raise $\left(\mathrm{h}_{\text {maxrate }}\right)$ and the magnitude of the corresponding lift coefficient, see Fig 6 and Fig 7, this is however attributed to aero-elastic deformation, as discussed later in this section. 


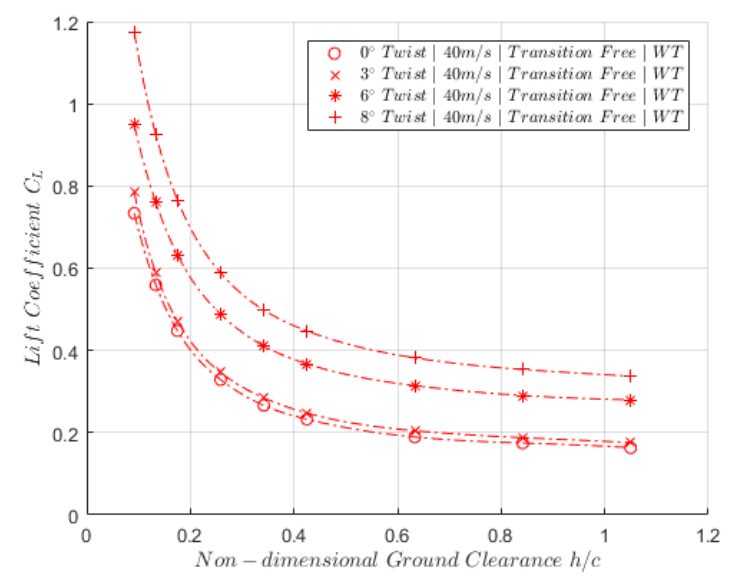

(a) Transition Free

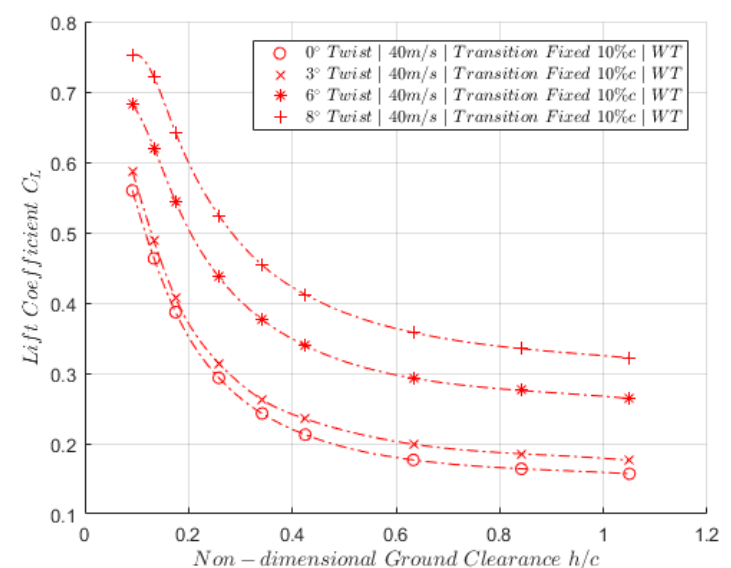

(b) Transition Fixed 10\% chord

Figure 6. Wind tunnel measurements at $R_{c}=320000$, both transition fixed and transition free, illustrating the effect of changes in both wing twist and ground clearance $(\mathrm{h} / \mathrm{c})$

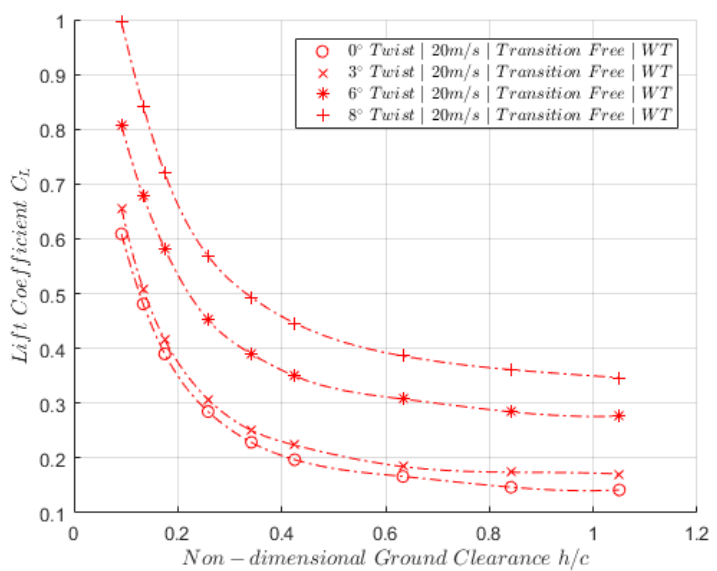

(a) Transition Free

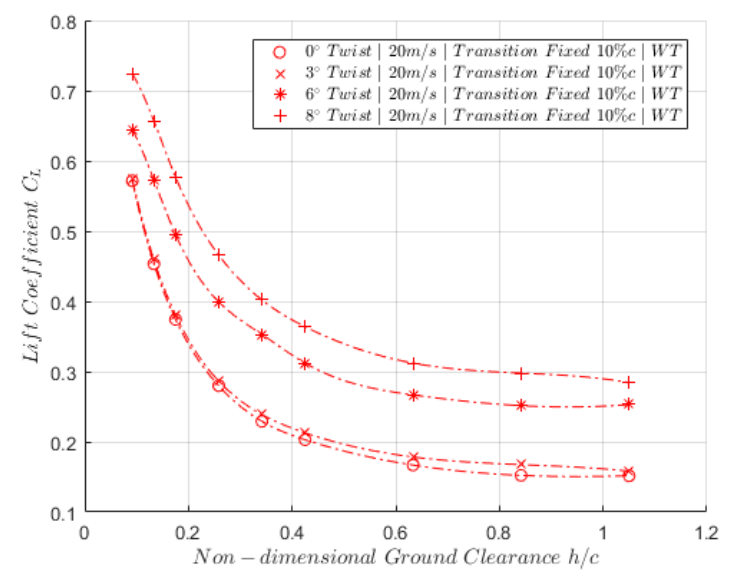

(b) Transition Fixed 10\% chord

Figure 7. Wind tunnel measurements at $R_{c}=160000$, both transition fixed and transition free, illustrating the effect of changes in both wing twist and ground clearance $(\mathrm{h} / \mathrm{c})$

Comparison between the RANS CFD simulations and wind tunnel measurements shows apparent correlation for the transition free wind tunnel wing configuration, see Fig 8(a). This was not as expected since the CFD simulation was carried out for a fully turbulent boundary layer (nominally corresponding to the transition fixed condition in the wind tunnel experiments). The difference between predicted and measured lift coefficient becomes more noticeable when the wing is in close ground proximity, $(\mathrm{h} / \mathrm{c}) \leq 0.4$, see Fig 8 (b); RANS CFD calculations appear to over-predict (-ve) lift coefficient in ground effect. Analysis of the CFD flow field simulation suggests that a possible reason for this difference could be the poor representation of the vortex formation/breakdown and the wing trailing edge separation region (plus any coupling between the two). The region of separation appears to be particularly sensitive when at low ground clearances as a result of the severity of the induced adverse pressure gradient at these test conditions. 


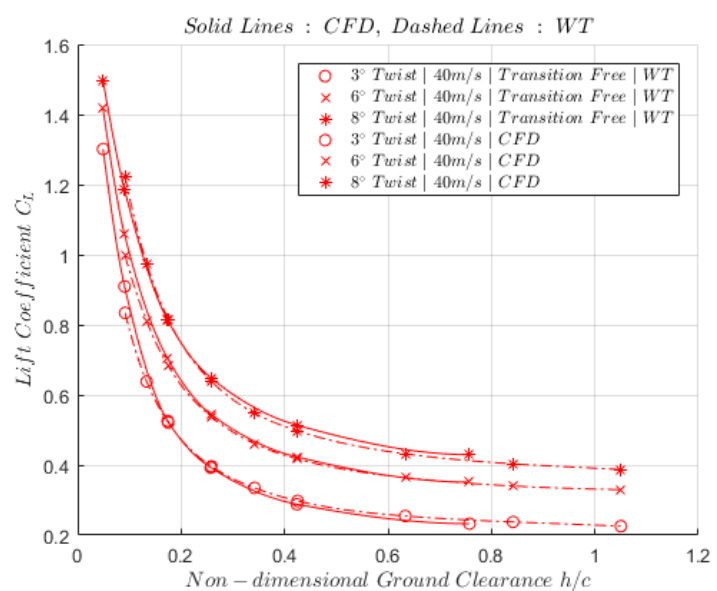

(a) Transition Free data from Wind Tunnel

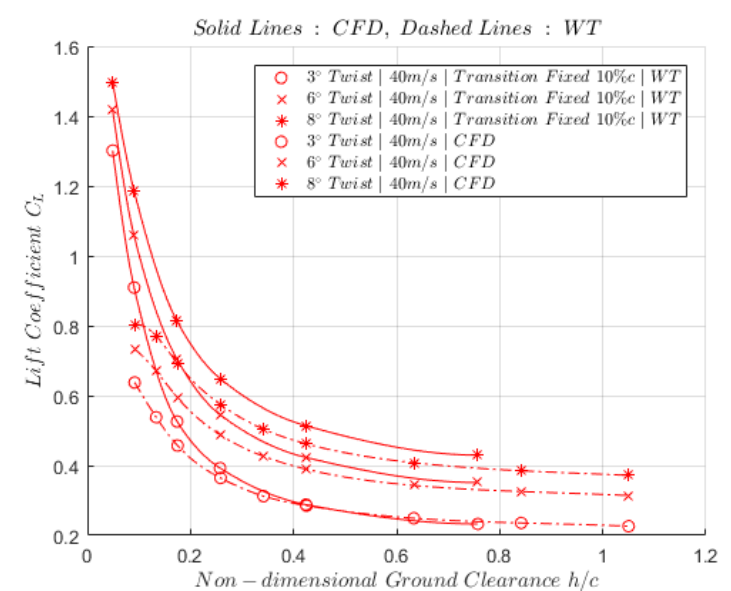

(b) Transition Fixed data from Wind Tunnel

Figure 8. A comparisson between the response of measured and predicted (-ve) lift coefficient to changes in ground clearance and wing twist.

\section{A. Assessment of tip vortex trajectory in close ground proximity}

The reduction in the slope of the (-ve) lift coefficient vs (h/c) data, seen in the wind tunnel data at very low ground clearances (see Fig 8(b)), has been attributed by a number of previous researchers (see for example Zhang et al [7], Zerihan et al [8]) to tip vortex instabilities coupled with trailing edge separation and vortex breakdown. The trajectory of the tip vortex, taken from the CFD simulations, see for example Fig 9, confirms the in-board migration of the vortex core and the sensitivity of this trajectory to ground clearance. As the wing-body height is reduced, tip vortex strength appears to increase, leading to the formation of a secondary vortex at low ground clearances, observed in the CFD simulations at heights approaching h/c=17\%, see Fig 9 and Fig 10. The higher vortex strength associated with the more highly twisted wing configurations also leads to more advanced secondary vortex formation. Both inboard displacement of the vortex core and the formation of a secondary vortex are believed to destabilize the primary tip vortex, this was however not observed in the steady RANS simulations.

The vortex core trajectory, at a given ground clearance, does not appear to be significantly affected by changes in wing twist. Vortex strength however increases with increasing twist angle, a higher wash-in twist angle tends to move the tip vortex more in-board. As would be expected, vertical displacements of the primary tip vortex were seen to be constrained by the proximity of the ground plane, see Fig 9, when compared to the corresponding out-of-ground effect vortex core path. 


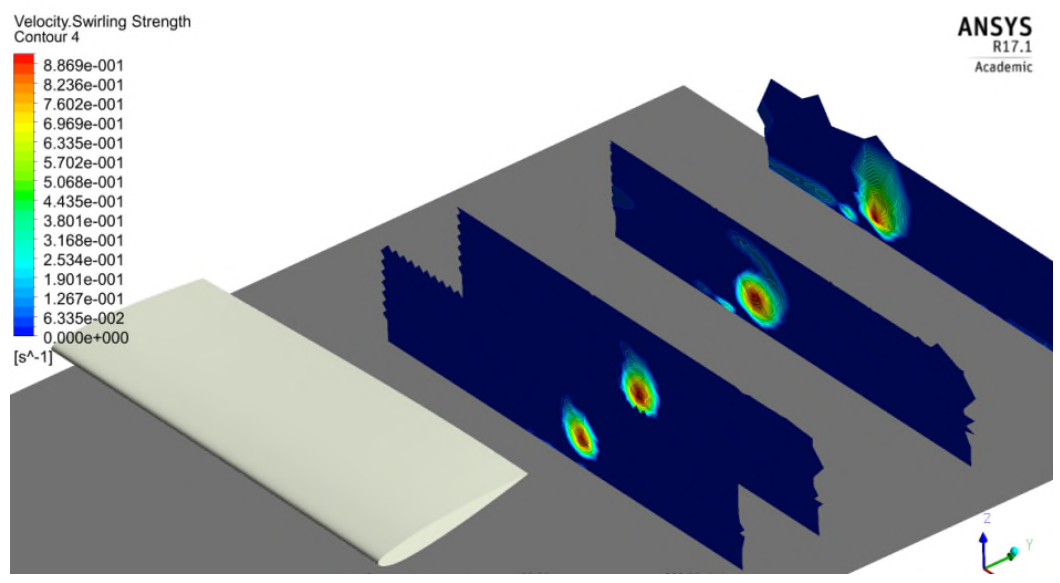

Figure 9. RANS CFD simulation of wing tip vortex trajectory (depicted by Velocity Swirling Strength). Wing configuration: $8^{\circ}$ twist, $R e / m \approx 2.8 \times 10^{6}, h / c=9 \%$

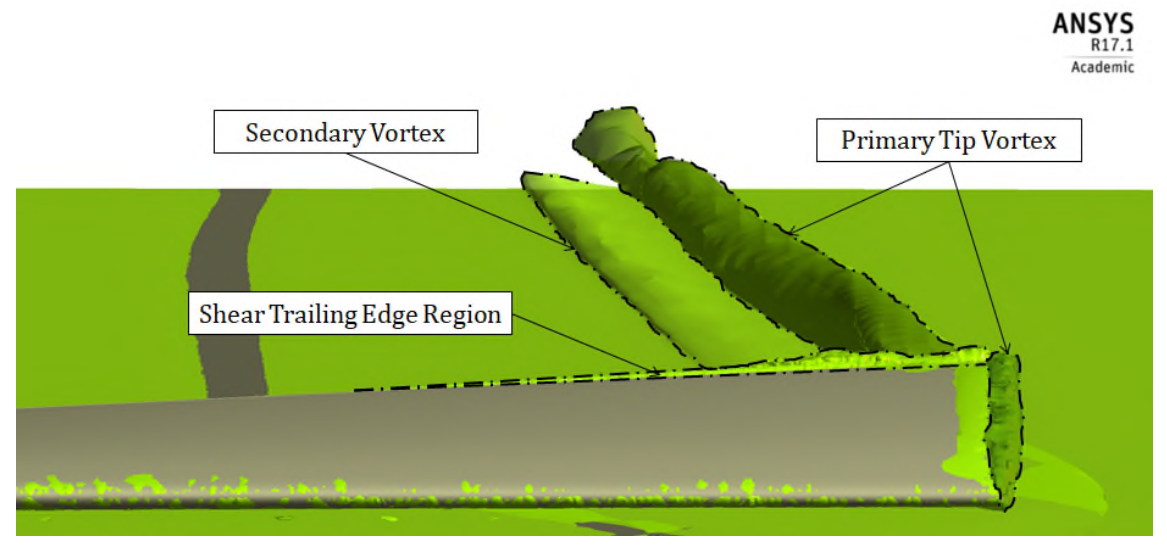

Figure 10. RANS CFD simulation illustrating different vorticity regions in the vicinity of a wash-in twisted wing in ground effect, depicted by helicity contours - view looking downwind, $8^{\circ}$ twist, Re/m $\approx 2.8 \times 10^{6}, h / c=$ $12.5 \%$

The RANS CFD prediction that the tip vortex core is displaced in-board with reducing ground clearance was only partially confirmed by the experimental data, Fig 11, due to the limited number of experimental data points. This limitation was attributed to a lack of fidelity in the tuft grid measuring technique and, as a result, further work with an optical flow visualisation approach is planned. Similarly, the existence of a secondary vortex on the ground plane, as predicted in some conditions by the CFD simulations, was not visualised by the tuft grid in the wind tunnel. 


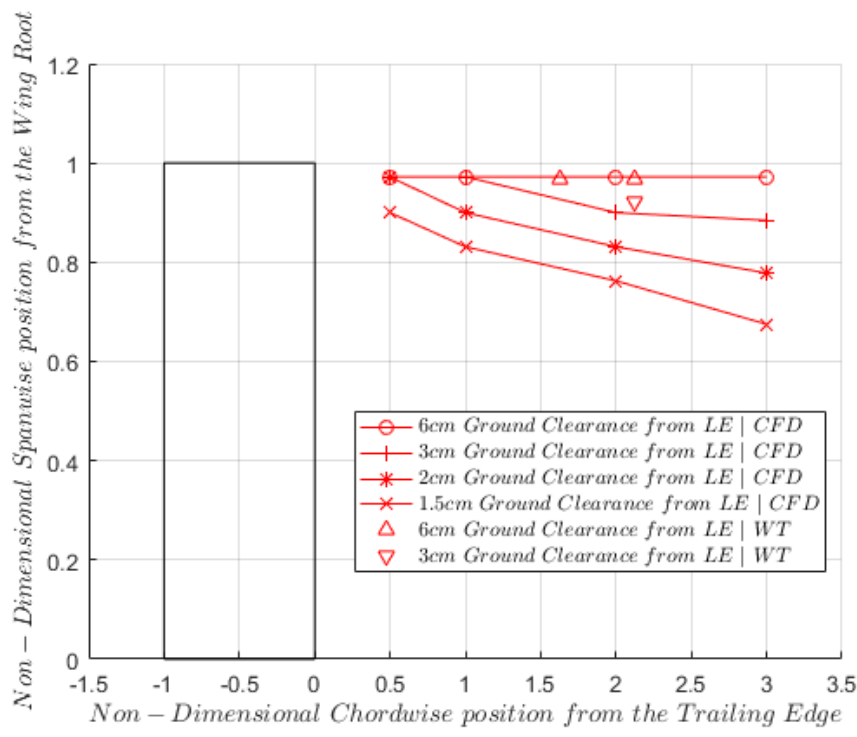

Figure 11. Comparison of RANS CFD predictions of wing tip vortex trajectory in the range $0.125<(\mathrm{h} / \mathrm{c})<0.5$, with experimental measurements at $(h / c)=0.25$ and 0.5 , for the $0^{\circ}$ twist wing.

\section{B. Effects of Aero-elastic deformation}

The wing models were CNC machined, from solid, using a composite tooling board, Necuron651, with an embedded cylindrical steel spar centred on the wing $1 / 4$ chord to increase bending stiffness. Static loading tests were undertaken on each $1 / 2$ wing mounted on the centre-body to establish tip bending stiffness and tip torsional stiffness (relative to the wing root). This data, summarised in Table 1, demonstrates the relative consistency in both torsional and bending stiffness between the four different wing models, facilitating comparison of the measured aerodynamic characteristics.

Table 1. Summary of the model elastic characteristics, in terms of torsional stiffness and bending stiffness (relative to the wing root), due to static loading applied at the tip.

\begin{tabular}{|c|c|c|}
\hline $\begin{array}{c}\text { wing model } \\
\text { configuration }\end{array}$ & $\begin{array}{c}\text { torsional } \\
\text { stiffness } \\
(\mathrm{deg} / \mathrm{Nm})\end{array}$ & $\begin{array}{c}\text { bending stiffness } \\
(\mathrm{m} / \mathrm{N})\end{array}$ \\
\hline & & $2.370 \mathrm{E}-04$ \\
\hline 0 twist & -0.434 & $2.369 \mathrm{E}-04$ \\
\hline 3 twist & -0.469 & $2.458 \mathrm{E}-04$ \\
\hline 6 twist & -0.471 & $2.429 \mathrm{E}-04$ \\
\hline 8 twist & -0.455 & \\
\hline
\end{tabular}

Measurements of wing deflection during the wind tunnel tests, using the VMD technique, showed the extent of the aero-elastic distortion at different ground clearances and different wing twist. Vertical displacements of up to $2.7 \mathrm{~mm}$ were recorded at the wing tip, coupled with a $1^{\circ}$ twist-back angle (see for example the data for the $8^{\circ}$ twist wing at $(\mathrm{h} / \mathrm{c})=8.3 \%, \mathrm{Re} / \mathrm{m}=2.8 \times 10^{6}$, shown in Fig. 12(c)). For that wing configuration, aero-elastically-induced vertical displacements account for a $27 \%$ decrease in ground clearance and $12.5 \%$ change in twist. Recalling the effect of changes in ground clearance on lift coefficient, see Figures 6 and 7, aero-elastic deformation is clearly a significant factor influencing the identification of the ground clearance at which both maximum (-ve) lift coefficient $\left(\mathrm{h}_{\text {maxlift }}\right)$ and maximum sensitivity of lift coefficient to ground clearance $\left(\mathrm{h}_{\text {maxrate }}\right)$ occurs. A stiffer wing could potentially displace both $h_{\text {maxlift }}$ and $h_{\text {maxrate }}$ to lower ground clearances. (Note: ground clearance is measured relative to the wing root, which is un-deflected, as opposed to the wing tip which is subject to aero-elastic distortions). 
Measured wing deflections increased with wind tunnel freestream dynamic pressure, as would be expected. High wing twist angles increase aero-elastic distortions for a given ground clearance and freestream flow velocity, see Fig. 13. Similar observations can be made with regard to Fig. 12 in which it can be seen that, at a given twist configuration and dynamic pressure, reducing ground clearance has the effect of increasing aero-elastically-induced displacements. Vertical displacements observed at the wing tip are directly related to the magnitude of the lift produced by the wing. As a result, and as free transition was seen to generate more lift than cases where the transition was fixed, free transition is thought to further increase aero-elastic distortion.

The aero-elastic deformation of the wing has the advantage of reducing the effective twist angle, which is beneficial for both drag coefficient and lift to drag ratio, see Fig 3 and 5.

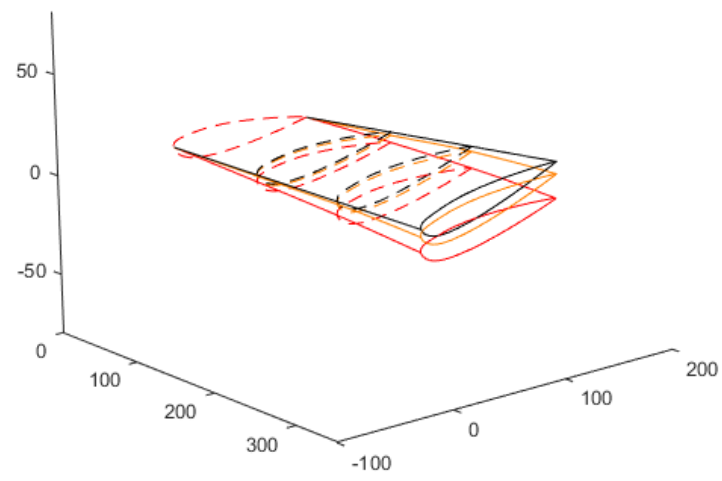

(a) $(h / c)=83 \%$

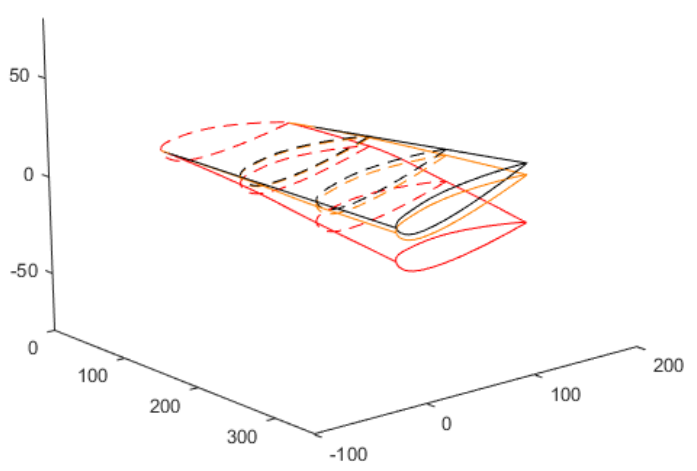

(b) $(h / c)=25 \%$

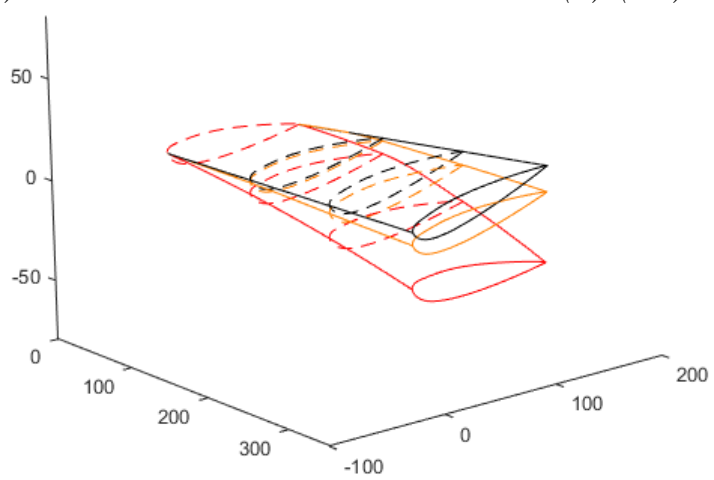

(c) $(h / c)=8.3 \%$

Figure 12. Aero-elastic deflections, measured using the VMD Technique, in the range $8.3 \%<($ h/c $)<83 \%$ highlighting the impact of ground clearance and run velocity for the $8^{\circ}$ twisted wing [black contours: undeformed wing - orange: $R e / m \approx 1.4 \times 10^{6}$, red: $\left.R e / m \approx 2.8 \times 10^{6}\right]$

NOTE : vertical displacements are exaggerated by a factor 10. 


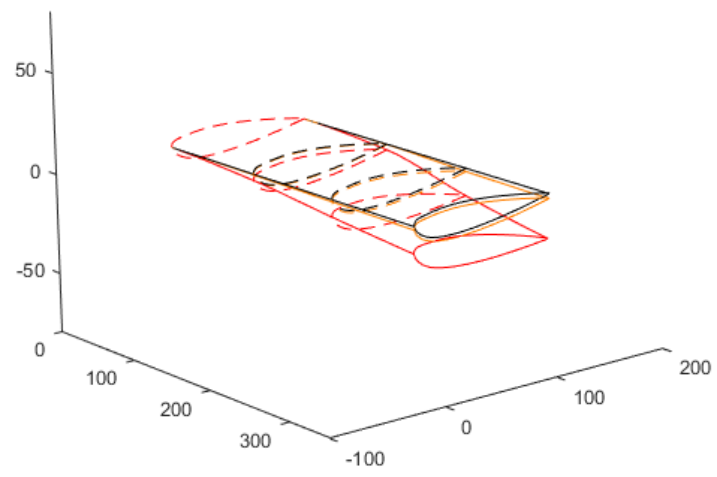

(a) Non-Twisted Wing

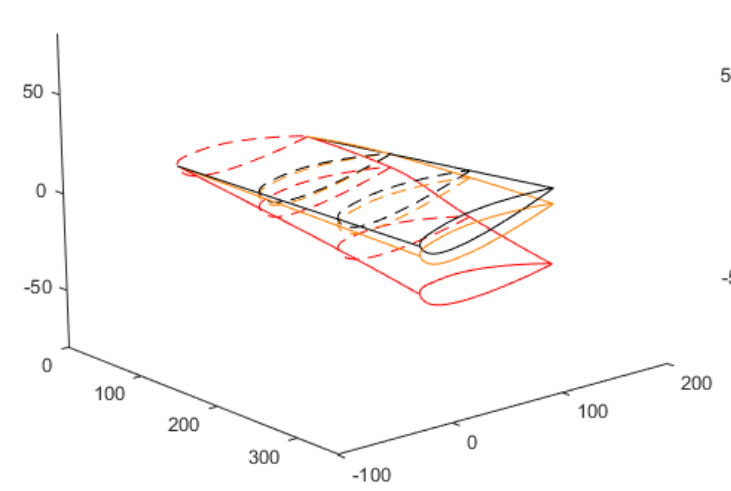

(c) $6^{\circ}$ Twist

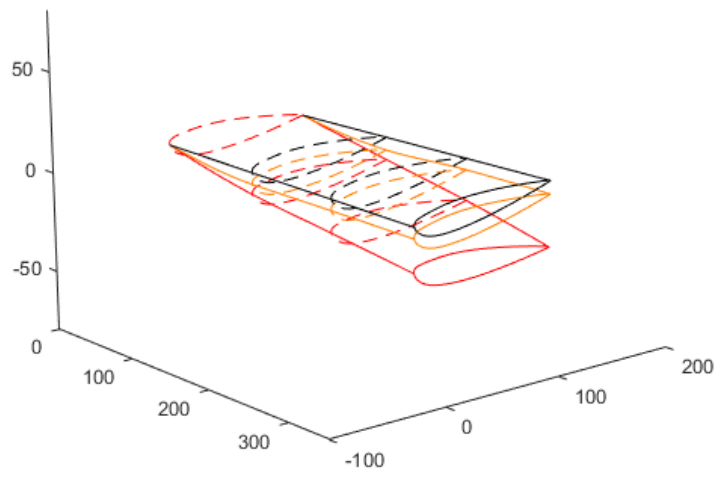

(b) $3^{\circ}$ Twist

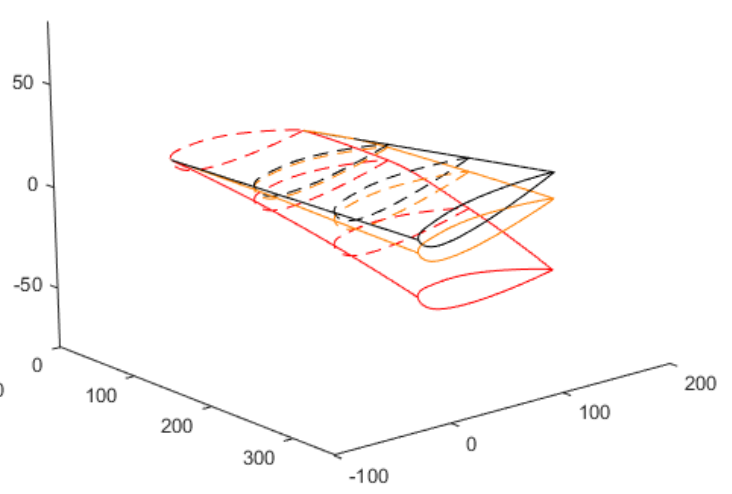

(d) $8^{\circ}$ Twist

Figure 13. Aero-elastic deflections measured using the VMD Technique in the range $0^{\circ}<$ Twist $<8^{\circ}$ at $h / c=$ 8.3\% [black contours: undeformed wing-orange: $R e / m \approx 1.4 \times 10^{6}, \mathrm{red}: \mathrm{Re} / \mathrm{m} \approx 2.8 \times 10^{6}$ ] NOTE: vertical displacements are amplified by a factor 10.

Model deflection data made it possible for an additional CFD simulation to be conducted on a 'true' (deformed) model geometry to facilitate a more realistic comparison with the experimental data, integrating both bending and torsion characteristics, see Fig 14. The wing configuration chosen for this comparison was the $8^{\circ}$ twist, h/c $=9 \%$, $R e / m \approx 2.8 \times 10^{6}$ case, which showed the biggest tip displacements during the wind tunnel test programme. 


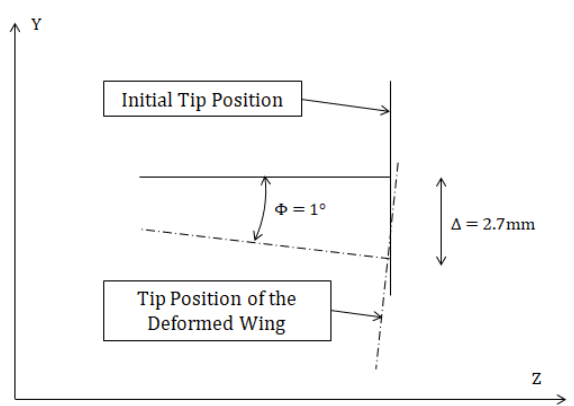

(a) Schematic view of near-tip region looking downwind

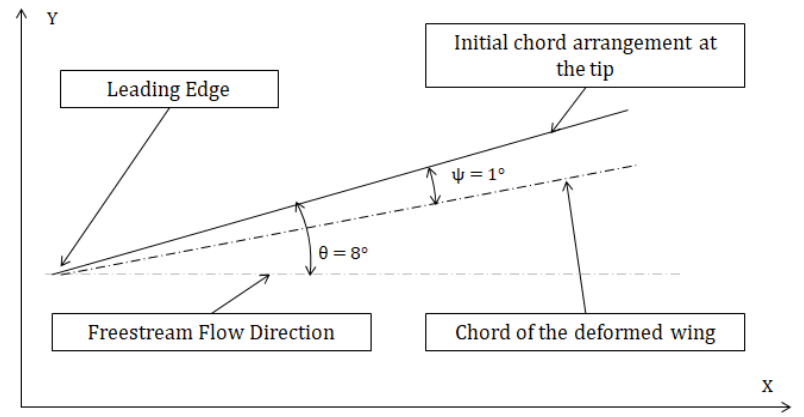

(b) view looking in-board (flow from left to right)

Figure 14. Schematic of 'under-load' wing geometry, compared with the initial un-deformed wing

The primary mesh and solver settings were kept identical to the initial 'undeformed wing' CFD simulations. The mesh was re-computed for the new (deflected) geometry, the number of cells was kept nominally the same (1.52 million). Comparing the rigid body and deflected RANS CFD predictions, the actual 'under-load' model produces up to $7.9 \%$ more (-ve) lift coefficient and $7.6 \%$ greater drag coefficient compared to the corresponding un-deformed wing configuration. Wing deflection appears to have very little effect on tip vortex strength but the trajectory of the tip vortex core appears to be more in-board, which is believed to increase the likelihood of vortex breakdown. As a result, high wing bending and torsion, associated with both the high Re test cases and high wing twist configurations, are responsible for a greater sensitivity of (-ve) lift coefficient to ground clearance.

\section{Unsteady Aero-elastic deformations}

At the lowest ground clearances tested $(0.05<(\mathrm{h} / \mathrm{c})<0.07)$, there is evidence of unsteady loads on the wing for the non-zero twist wing configurations. This was apparent for both the transition fixed and transition free test conditions, see Fig 7. This characteristic has previously been attributed to vortex burst close to the maximum (-ve) lift coefficient ground clearance, see Zhang et al.[7] Heights at which dynamic oscillations arose, see Table 2, effectively correspond to heights at which maximum (-ve) lift coefficient was recorded, see Fig 6, referred to here as the vortex bursting height.

It is postulated that, when the tip vortex bursts, some of the vortex-induced tip suction is suppressed and the upwash associated with the tip vortex is reduced. This, in turn, enlarges the separated region at the trailing edge towards the tip which has the effect of reducing (-ve) lift coefficient and increasing drag coefficient. As a result of the reduction in (-ve) lift, the wing displaces away from the ground (due the aero-elastic stiffness), eventually reaching a ground clearance at which the tip vortex can reform. This cyclic process produces periodic oscillation of the wing tip (in both heave and pitch angle) which is damped at ground clearances above that of the maximum measured lift coefficient $\left(\mathrm{h}_{\text {maxlift}}\right)$. Unsteady loads were such as to prevent the use of the VMD measurement technique at these unsteady conditions using conventional cameras (acquisition rate $29 \mathrm{~Hz}$ ) and further work with high speed cameras is required to understand the motion.

Table 2. Summary of the wing configurations for which aero-elastically-induced oscillations of the wing tip were observed during wind tunnel testing

\begin{tabular}{|c|c|c|c|}
\hline $\begin{array}{l}\text { Twist } \\
\text { Configuration }\end{array}$ & $\begin{array}{l}\text { Freestream flow } \\
\text { Velocity }\end{array}$ & Ground Clearance & Transition \\
\hline $3^{\circ}$ & $40 \mathrm{~m} / \mathrm{s}$ & $5 \mathrm{~mm}$ & Free \\
\hline $6^{\circ}$ & $40 \mathrm{~m} / \mathrm{s}$ & $5 \mathrm{~mm}$ & Fixed @ $10 \% \mathrm{c}$ \\
\hline $8^{\circ}$ & $40 \mathrm{~m} / \mathrm{s}$ & $7.5 \mathrm{~mm}$ & Fixed @ $10 \% \mathrm{c}$ \\
\hline
\end{tabular}




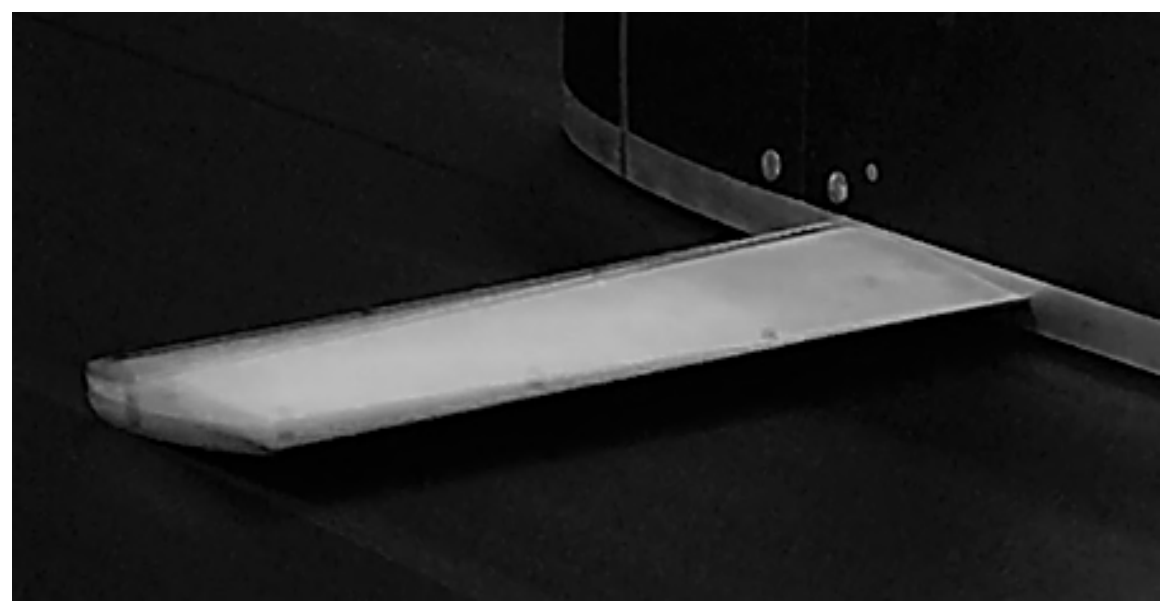

Figure 15. Long-exposure photograph of the $8^{\circ}$ twisted wing illustrating the dynamic aero-elastic oscillations of the tip in close ground proximity.

\section{Conclusions}

The aerodynamic characteristic of an elastic, inverted twisted wing in close ground proximity was studied using both a RANS CFD simulation and moving ground wind tunnel tests. The general trend, as was expected from previously published data, is for both (-ve) lift coefficient and drag coefficient to increase with reducing ground clearance. Increasing wash-in twist has the adverse effect of limiting the benefits of ground proximity in terms of (-ve) lift coefficient generation, whilst at the same time, amplifying the adverse effect of ground proximity on drag coefficient. As a consequence wing lift to drag ratio is reduced. The aerodynamic characteristics close to the ground are primarily dependent on the extent of the region of separated flow near the wing trailing edge and the tip vortex characteristics, which are linked. In very close proximity to the ground (typically $\mathrm{h} / \mathrm{c}<10 \%$ ), the tip vortex appears to burst, suppressing the induced up-wash flow component near the tip which normally helps to maintain attached flow on the outboard region of the wing. The reason for tip vortex breakdown is believed to be due to a number of sources which includes (i) in-board migration of the vortex core and (ii) formation of a secondary vortex, both of which are associated with low ground clearances.

Wind tunnel measurements, using videogrammetry techniques, have shown the extent of aero-elastic deformation in ground effect. Significant vertical displacements were recorded, accounting for up to $27 \%$ of the actual ground clearance and a $12.5 \%$ 'un-twisting' of the wing, at the tip. Aero-elastic deformation is seen to accentuate the inboard trajectory of the tip vortex, which is believed to trigger vortex breakdown at a higher ground clearance than that at which it would occur for a rigid (un-deformed) wing.

When the tip vortex bursts, which normally occurs at the maximum (-ve) lift condition, dynamic oscillations of the wing tip were recorded. Oscillations were seen to be neutrally stable, incorporating both heave and pitching motions. Those oscillations ceased immediately when the wing was raised to higher ground clearances, where the tip vortex is reformed.

\section{References}

[1] Barrows, D.A. Videogrammetric Model Deformation measurement technique for wind tunnel applications. 45th AIAA Aerospace Sciences Meeting and Exhibit. AIAA 2007-1163 (2007) https://doi.org/10.2514/6.2007-1163

[2] Barzic, J-B. The aerodynamic effect of twist for inverted wings in very close ground proximity. MSc Thesis. Cranfield University UK (2017) 
[3] Burner, A.W. and Liu, T. Videogrammetric Model Deformation measurement technique. Jn of Aircraft 38 (2001), pp. 745754.

[4] Doig D., Barber T.J. and Neely A.J. The Influence of compressibility on the aerodynamics of an inverted wing in ground effect. Jn. of Fluids Engineering Vol33. No6 (2011). doi:10.1115/1.4004084

[5] Hunsaker, D.F., Philips, W.F. and Joo, J.J. Designing wings with fixed twist for minimum induced drag. 55th AIAA Aerospace Sciences Meeting AIAA 2017-1419 (2017), https://doi.org/10.2514/6.2017-1419

[6] Lane, K.A., Marshall, D.D. and McDonald, R.A. Lift Superposition and Aerodynamic Twist Optimization for Achieving Desired Lift Distributions. 48 ${ }^{\text {th }}$ AIAA Aerospace Sciences Meeting. AIAA 2010-1227 (2010) https://doi.org/10.2514/6.2010$\underline{1227}$

[7] Zhang, X., Zerihan, J., Ruhrman, A. And Deviese, M. Tip Vortices generated by a Wing in Ground Effect. MA Thesis. University of Southampton 2002.

[8] Zerihan, J., Zhang X., A Single Element Wing in Ground Effect; Comparisons of Experiments and Computation. 39 ${ }^{\text {th }}$ AIAA Aerospace Sciences Meeting. AIAA 2001-1250 (2001), https://doi.org/10.2514/6.2001-423 


\section{Cranfield University}

2019-01-06

\section{A study of an aero-elastic twisted inverted wing in close ground proximity}

Barzic, Jean-Baptiste

American Institute of Aeronautics and Astronautics

https://dspace.lib.cranfield.ac.uk/handle/1826/13889

Downloaded from Cranfield Library Services E-Repository 